\title{
Significance of De Ritis (Aspartate Transaminase/Alanine Transaminase) Ratio as a Significant Prognostic But Not Predictive Biomarker in Japanese Patients with Metastatic Castration-resistant Prostate Cancer Treated with Cabazitaxel
}

\author{
HIDEAKI MIYAKE, YUTO MATSUSHITA, HIROMITSU WATANABE, KEITA TAMURA, TAKAHISA SUZUKI, \\ DAISUKE MOTOYAMA, TOSHIKI ITO, TAKAYUKI SUGIYAMA and ATSUSHI OTSUKA
}

Department of Urology, Hamamatsu University School of Medicine, Hamamatsu, Japan

\begin{abstract}
Background/Aim: To date, there have not been any established biomarkers predicting the efficacy of cabazitaxel in patients with metastatic castration-resistant prostate cancer ( $m C R P C)$. The objective of this study was to evaluate the significance of the aspartate aminotransaminase (AST)/alanine aminotransaminase (ALT) ratio (De Ritis ratio) as a biomarker for $m C R P C$ patients receiving cabazitaxel. Patients and Methods: This study included 74 consecutive docetaxel-refractory $m C R P C$ patients treated with cabazitaxel. It assessed the impact of the pretreatment De Ritis ratio, in addition to conventional clinicopathological parameters, on the oncological outcomes in these patients. Results: After treatment with cabazitaxel, $22(29.7 \%)$ of the 74 patients achieved a prostate-specific antigen (PSA) response; however, there was no significant difference in the PSA response rate between patients with a low De Ritis ratio $(<1.35)$ and those with a high ratio $(\geq 1.35)$. In this series, the median periods of PSA progression-free survival (PFS) and overall survival (OS) after the introduction of cabazitaxel were 4.2 and 14.7 months, respectively. No significant difference was noted in PSA PFS between the low and high De Ritis ratio groups, whereas $O S$ in the high De Ritis ratio group was significantly poorer compared with that in the low De Ritis ratio group. Univariate analysis showed the significant impact of the De Ritis ratio on OS, but not PFS, in these 74 patients. Furthermore, the De Ritis ratio, in addition to the
\end{abstract}

Correspondence to: Hideaki Miyake, Department of Urology, Hamamatsu University School of Medicine, 1-20-1 Handayama, Higashi-Ku, Hamamatsu 431-3192, Japan. Tel: +81 534352306, Fax: +81 534352305, e-mail: hideakimiyake@hotmail.com

Key Words: Castration-resistant prostate cancer, De Ritis ratio, cabazitaxel, biomarker. performance status and lactate dehydrogenase level, was shown to be independently associated with OS on multivariate analysis. Conclusion: Assessment of the De Ritis ratio may provide useful prognostic, but not predictive, information on cabazitaxel therapy in $\mathrm{mCRPC}$ patients.

Until recently, docetaxel was regarded as the only standard therapeutic option for patients with metastatic castrationresistant prostate cancer (mCRPC). During the last few years, however, multiple new agents with different mechanisms of action has been demonstrated to have a prognostic benefit in mCRPC patients showing disease progression after treatment with docetaxel $(1,2)$. Of these, cabazitaxel has been widely accepted as a next-generation systemic chemotherapy for mCRPC based on the outcomes of the pivotal phase III TROPIC trial, which was conducted targeting docetaxel-refractory mCRPC patients and showed the significant prognostic advantage of cabazitaxel compared with mitoxantrone (3). Despite the excellent antitumor activity of cabazitaxel, the optimal sequential therapy for mCRPC patients after the failure of docetaxel is still unclear, since new androgen receptor-axis-targeted (ARAT) agents, abiraterone acetate (AA) and enzalutamide (Enz), were simultaneously introduced as promising agents against docetaxel-refractory mCRPC $(4,5)$, and no clinical trials have directly compared patterns of sequential therapy in a post-chemotherapy setting. Collectively, these findings suggest that it is necessary to identify biomarkers closely reflecting the oncological outcomes using these agents, particularly cabazitaxel, to determine appropriate candidates for post-docetaxel treatment with each agent.

Aminotransaminases, including aspartate aminotransaminase (AST) and alanine aminotransaminase (ALT), are enzymes released from liver cells into the bloodstream, reflecting the degree of hepatocellular damage, Therefore, they have been used as part of the commonly requested panel for assessment 
of liver function in routine clinical practice (6). In 1957, De Ritis et al initially reported the ratio of serum activities of AST and ALT as one of the diagnostic markers for viral hepatitis (7), known as the De Ritis ratio thereafter. Despite originally being proposed as a parameter of acute viral hepatitis, this ratio has subsequently been shown to be a useful biomarker associated with the prognosis of patients with several types of malignant tumor (8-11). Recently, we have also reported the independent impact of the preoperative De Ritis ratio on extravesical recurrence-free survival in 109 patients with upper urinary tract urothelial carcinoma who underwent nephroureterectomy (12). To date, however, no study has investigated the impact of the De Ritis ratio on oncological outcomes in mCRPC patients.

Considering these findings, a total of 74 docetaxelrefractory mCRPC patients who were treated with cabazitaxel were retrospectively included in this study and the prognostic, as well as predictive values of the baseline De Ritis ratio assessed prior to the introduction of cabazitaxel in these patients was analyzed.

\section{Patients and Methods}

Patients. This study was conducted by retrospectively reviewing clinicopathological data from a total of 74 Japanese mCRPC patients who were diagnosed with progressive disease during or after treatment with a doctaxel-containing regimen and subsequently received cabazitaxel therapy between September 2014 and June 2017 in a routine clinical setting. All the patients included in this study were judged to have prostatic adenocarcinoma by histopathological examination and were initially treated with androgen-deprivation therapy (ADT), involving medical or surgical castration. According to either the Prostate Cancer Working Group 2 (PCWG2) criteria (13) or the Response Evaluation Criteria in Solid Tumors (14), disease progression against primary ADT, indicating the emergence of CRPC, was defined as prostate-specific antigen (PSA) or radiographic progression, respectively, in patients maintaining a serum testosterone level $<50 \mathrm{ng} / \mathrm{dl}$.

Treatments. Following the failure of the primary ADT, several patterns of sequential therapy using ARAT agents and/or docetaxel were provided based on the preference of the physicians without strictly regulated criteria; thus, prior to the cabazitaxel therapy, 59 patients (79.8\%) received AA and/or Enz in addition to docetaxel, and the remaining $15(20.2 \%)$ were treated with docetaxel alone. As a rule, ARAT agents and cabazitaxel were administered according to the standard dosing schedules, as previously reported [3-5], whereas docetaxel was generally given at a dose of $70 \mathrm{mg} / \mathrm{m}^{2}$ every 3 weeks considering its severe toxic profile for Japanese patients (15). The treatment with either agent was continued until the occurrence of disease progression, assessed based on the same definition as applied to the primary ADT. In patients developing treatment-associated adverse events (AEs) corresponding to grade $\geq 3$, dose modification and/or treatment delay of either agent was permitted.

Evaluation. The clinicopathological data of each patient were obtained from the medical records. Before introducing cabazitaxel, the Eastern Cooperative Oncology Group (ECOG) performance status (PS) was assessed, and the number and location of metastatic lesions were evaluated by radiological examinations using computed tomography and radionuclide bone scans. All laboratory data, including hemoglobin $(\mathrm{Hb})$, albumin (Alb), lactate dehydrogenase (LDH), PSA, AST and ALT levels, were also measured in each patient by standard clinical testing methods prior to the introduction of cabazitaxel. After the administration of cabazitaxel, the PSA level in addition to bone marrow, renal and liver functions were measured every 4-6 weeks, while radiological examinations were performed based on the discretion of the treating physicians.

Statistical analysis. All statistical analyses were performed using Statview 5.0 software (Abacus Concepts, Inc., Berkley, CA, USA), and a $p$-Value $<0.05$ was considered significant. Differences of several parameters between the two groups were analyzed by the unpaired $t$-test and Chi-squared test. PSA progression-free survival (PFS) and overall survival (OS) rates were calculated by the Kaplan-Meier method, and differences were determined by the logrank test. The associations of certain factors with PSA PFS and OS were analyzed using the Cox proportional hazards regression model.

\section{Results}

Characteristics of included patients according to De Ritis ratio. The median value of the AST/ALT ratio (De Ritis ratio) assessed prior to the introduction of cabazitaxel in the 74 mCRPC patients included in this study was 1.35 , which was used as the cutoff point of this ratio. Table I summarizes the clinicopathological characteristics of these 74 patients according to the De Ritis ratio. There were significant differences in age, LDH level, PSA level and proportion of patients with bone metastasis between the low and high De Ritis ratio groups; however, no significant differences in the remaining factors were noted between the two groups.

Oncological outcomes according to De Ritis ratio. In this series, the median cycles of cabazitaxel therapy were 5 (range=1-12 cycles). Table II shows the reduction patterns of the serum PSA values during treatment with cabazitaxel therapy according to the De Ritis ratio. Following treatment with cabazitaxel, $>0 \%$ PSA decline was observed in 47 patients $(63.5 \%)$, and $35(47.3 \%)$ and $22(29.7 \%)$ achieved a PSA decline $\geq 30 \%$ and $\geq 50 \%$ from the baseline, respectively. As shown in Table II, there were no significant differences in the proportions of patients achieving PSA decline $>0, \geq 30$ and $\geq 50 \%$ between the low and high De Ritis ratio groups.

During the observation period of this study (median, 14 months; range $=2-29$ months), PSA progression occurred in 60 patients $(81.1 \%)$, and $41(55.4 \%)$ died. The median PSA PFS and OS periods of the 74 patients were 4.2 and 14.7 months, respectively (Figure 1). As shown in Figure 2, the median durations of PSA PFS in the low and high De Ritis ratio groups were 4.0 and 5.1 months, respectively, and there was no significant difference in the PSA PFS between the 
Table I. Characteristics of metastatic castration-resistant prostate cancer patients receiving cabazitaxel according to De Ritis ratio.

\begin{tabular}{|c|c|c|c|}
\hline \multirow[b]{2}{*}{ Variables } & \multicolumn{2}{|c|}{ De Ritis ratio } & \multirow[b]{2}{*}{$p$-Value } \\
\hline & $\leq 1.35(\mathrm{n}=37)$ & $>1.35(\mathrm{n}=37)$ & \\
\hline Median age (years, range) & $67.4(55-74)$ & $72.1(59-82)$ & 0.037 \\
\hline Median duration of ADT (months, range) & $18.9(6-108)$ & $18.4(3-90)$ & 0.19 \\
\hline ECOG performance status $(\%)$ & & & 0.53 \\
\hline 0 or 1 & $32(86.5)$ & $30(81.1)$ & \\
\hline$\geq 2$ & $5(13.5)$ & $7(18.9)$ & \\
\hline Symptom $(\%)$ & & & 0.5 \\
\hline Negative & $33(89.2)$ & $31(83.8)$ & \\
\hline Positive & $4(10.8)$ & $6(16.2)$ & \\
\hline Median value of baseline hemoglobin ( $\mathrm{g} / \mathrm{dl}$, range) & $12.1(8.2-14.7)$ & $11.6(7.3-14.3)$ & 0.3 \\
\hline Median value of baseline albumin (mg/dl, range) & $3.8(3.0-8.3)$ & $3.6(3.1-7.7)$ & 0.61 \\
\hline Median value of baseline LDH (IU/1, range) & $234.5(181.4-392.3)$ & $357.3(197.7-467.3)$ & 0.016 \\
\hline Median value of baseline ALP (IU/1, range) & $392.7(160.4-1182.3)$ & $433.0(177.7-2274.3)$ & 0.094 \\
\hline Median value of baseline PSA (ng/ml, range) & $72.3(6.4-450.3)$ & $83.6(8.7-701.2)$ & 0.041 \\
\hline Gleason score* $(\%)$ & & & 0.36 \\
\hline$\leq 7$ & $8(21.6)$ & $5(13.5)$ & \\
\hline$\geq 8$ & $29(78.4)$ & $32(86.5)$ & \\
\hline Bone metastasis $(\%)$ & & & 0.042 \\
\hline Negative & $15(40.5)$ & $7(18.9)$ & \\
\hline Positive & $22(59.5)$ & $30(81.1)$ & \\
\hline Lymph node metastasis $(\%)$ & & & 0.64 \\
\hline Negative & $22(59.5)$ & $20(54.1)$ & \\
\hline Positive & $15(40.5)$ & $17(45.9)$ & \\
\hline Visceral metastasis $(\%)$ & & & 0.76 \\
\hline Negative & $31(83.8)$ & $30(81.1)$ & \\
\hline Positive & $6(16.2)$ & $7(18.9)$ & \\
\hline
\end{tabular}

*Findings on biopsies performed prior to primary ADT. ADT: Androgen deprivation therapy; ECOG: Eastern Cooperative Oncology Group; LDH: lactate dehydrogenase; ALP: alkaline phosphatase; PSA: prostate-specific antigen.

two groups; however, the OS in the high De Ritis ratio group (median, 13.9 months) was significantly inferior to that in the low De Ritis ratio group (median, 16.1 months).

Assessment of prognostic parameters. The association of several clinicopathological factors with the PSA PFS and OS in the 74 patients was then evaluated (Table III). Univariate analysis identified the following significant prognostic predictors: the ECOG PS, clinical symptoms and LDH level for PSA PFS, and the ECOG PS, clinical symptoms, Hb level, LDH level and De Ritis ratio for OS. Furthermore, on multivariate analyses of these significant parameters, the following factors were shown to have independent impacts: ECOG PS on PSA PFS, and ECOG PS, LDH level and De Ritis ratio on OS.

\section{Discussion}

Cabazitaxel, a second-generation taxane, has been widely regarded as one of the standard agents to treat mCRPC patients showing disease progression during or after
Table II. Prostate-specific antigen (PSA) decline from baseline prior to cabazitaxel therapy for castration-resistant prostate cancer according to De Ritis ratio.

\begin{tabular}{lcccc}
\hline & \multicolumn{3}{c}{ De Ritis ratio } \\
\cline { 2 - 4 } & Total & $\begin{array}{c}\leq 1.35 \\
(\mathrm{n}=74)\end{array}$ & $\begin{array}{c}1.35< \\
(\mathrm{n}=37)\end{array}$ & $\begin{array}{c}p \text {-Value } \\
(\mathrm{n}=37)\end{array}$ \\
\hline PSA decline $>0 \%(\%)$ & $47(63.5)$ & $25(67.6)$ & $22(59.5)$ & 0.47 \\
PSA decline $\geq 30 \%(\%)$ & $35(47.3)$ & $19(51.4)$ & $16(43.2)$ & 0.48 \\
PSA decline $\geq 50 \%(\%)$ & $22(29.7)$ & $13(35.1)$ & $9(24.3)$ & 0.31 \\
\hline
\end{tabular}

treatment with docetaxel $(1,2)$, since the TROPIC trial demonstrated that cabazitaxel significantly prolonged the OS in patients with docetaxel-refractory mCRPC compared with mitoxantrone. It reduced the relative risk of death by $30 \%$ (median OS period, 15.1 versus 12.7 months, respectively; hazard ratio, 0.70) (3). In Japan as well, favorable disease control of mCRPC patients by cabazitaxel has been reported 

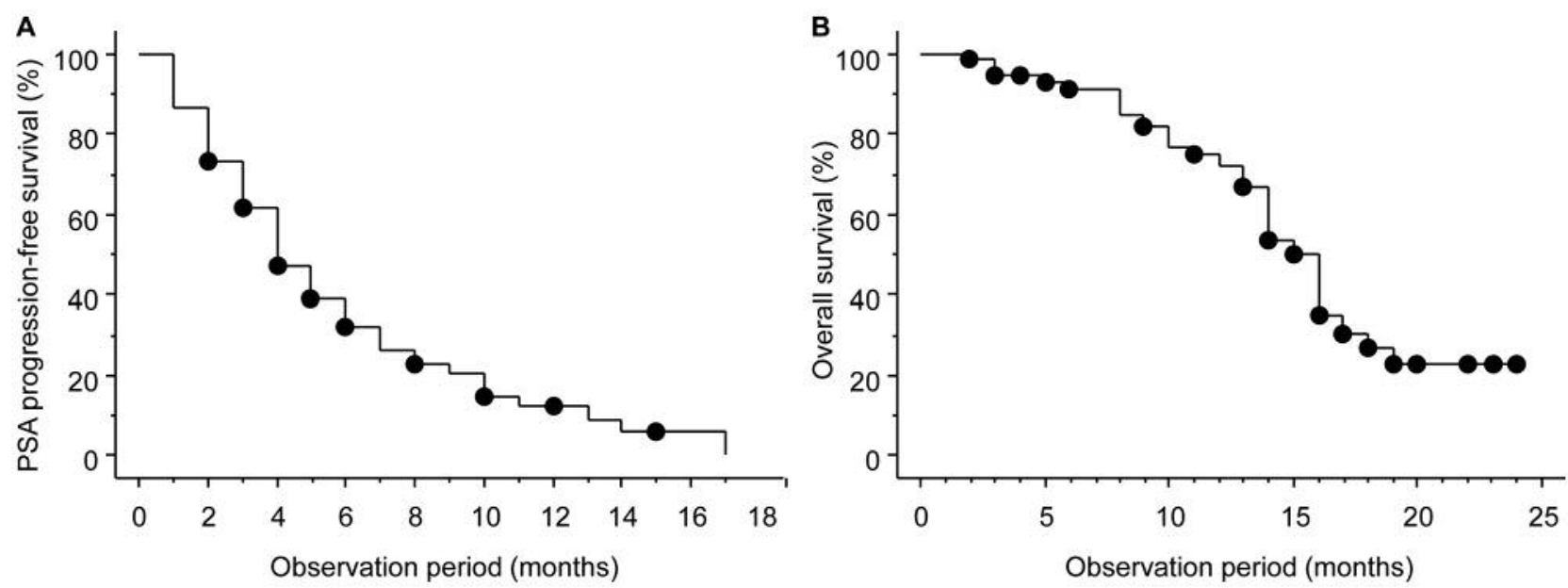

Figure 1. Prognostic outcomes in the overall patient cohort. A: Prostate-specific antigen progression-free survival in 74 metastatic castrationresistant prostate cancer ( $m C R P C)$ patients following cabazitaxel therapy. B: Overall survival in $74 m C R P C$ patients following cabazitaxel therapy.
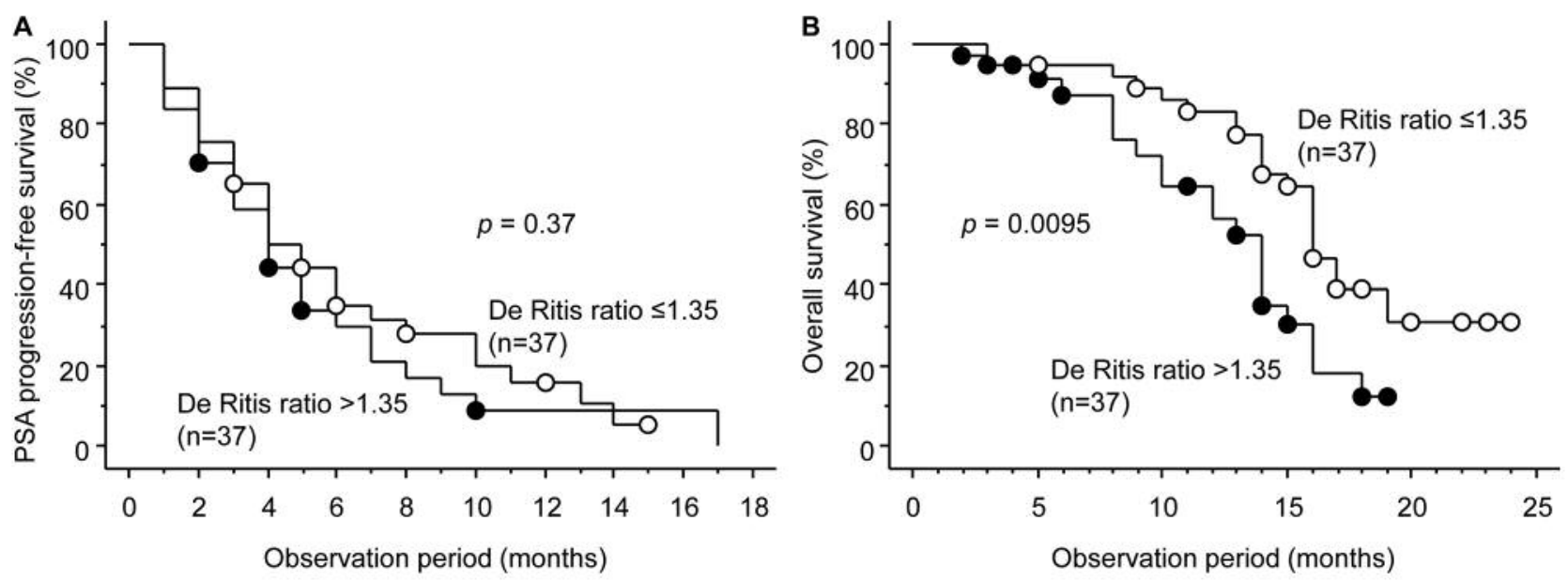

Figure 2. Prognostic outcomes according to the De Ritis ratio. A: Prostate-specific antigen progression-free survival in 74 metastatic castrationresistant prostate cancer ( $m C R P C$ ) patients following cabazitaxel therapy according to the De Ritis ratio. B: Overall survival in $74 m C R P C$ patients following cabazitaxel therapy according to the De Ritis ratio.

in both clinical trials and routine clinical practice; however, the ability of Japanese patients to tolerate cytotoxic chemotherapy, including cabazitaxel, was shown to be generally lower than that of Western populations (16-18). Moreover, there is limited information available on the development of biomarkers monitoring the clinical course of mCRPC patients receiving cabazitaxel (19). Considering these findings, we retrospectively assessed the oncological outcomes in a total of 74 docetaxel-refractory mCRPC patients who were treated with cabazitaxel, focusing on whether the De Ritis ratio could be used as a predictive as well as prognostic biomarker in these patients.

To date, there have been various studies showing the usefulness of aminotransaminases as prognostic factors in patients with malignant tumors, irrespective of the presence of liver-specific disease (8-11); however, since AST is widely expressed in various tissue types, but ALT is recognized as being more liver-specific (20), pathological 
Table III. Uni- and multivariate analyses of impacts of various parameters on prostate-specific antigen progression-free survival (PSA PFS) and overall survival $(O S)$ after introduction of cabazitaxel for castration-resistant prostate cancer patients.

\begin{tabular}{|c|c|c|c|c|c|c|c|c|}
\hline \multirow[b]{3}{*}{ Variables* } & \multicolumn{4}{|c|}{ PSA PFS } & \multicolumn{4}{|c|}{ OS } \\
\hline & \multicolumn{2}{|c|}{ Univariate analysis } & \multicolumn{2}{|c|}{ Multivariate analysis } & \multicolumn{2}{|c|}{ Univariate analysis } & \multicolumn{2}{|c|}{ Multivariate analysis } \\
\hline & HR & $p$-Value & HR & $p$-Value & HR & $p$-Value & HR & $p$-Value \\
\hline Age (years) $(\leq 69.0$ versus $>69.0)$ & 0.82 & 0.67 & - & - & 0.56 & 0.11 & - & - \\
\hline Duration of ADT (years) $(\leq 1.4$ versus $>1.4)$ & 1.43 & 0.19 & - & - & 1.29 & 0.44 & - & - \\
\hline ECOG performance status ( 0 or 1 versus $\geq 2$ ) & 0.27 & 0.018 & 0.34 & 0.042 & 0.22 & 0.015 & 0.27 & 0.039 \\
\hline Clinical symptoms (negative versus positive) & 0.29 & 0.024 & 0.68 & 0.39 & 0.26 & 0.038 & 0.66 & 0.38 \\
\hline Baseline hemoglobin $(\mathrm{g} / \mathrm{dl})(\leq 11.8$ versus $>11.8)$ & 1.34 & 0.42 & - & - & 2,28 & 0.04 & 1.59 & 0.28 \\
\hline Baseline albumin $(\mathrm{mg} / \mathrm{ml})(\leq 3.7$ versus $>3.7)$ & 1.14 & 0.56 & - & - & 1.28 & 0.32 & - & - \\
\hline Baseline LDH (IU/1) $(\leq 290$ versus $>290)$ & 0.21 & 0.017 & 0.56 & 0.2 & 0.19 & 0.0072 & 0.24 & 0.012 \\
\hline Baseline ALP (IU/l) ( $\leq 412$ versus $>412)$ & 0.57 & 0.21 & - & - & 0.58 & 0.22 & - & - \\
\hline Baseline De Ritis ratio $(\leq 1.35$ versus $>1.35)$ & 0.64 & 0.27 & - & - & 0.21 & 0.011 & 0.3 & 0.042 \\
\hline Baseline PSA $(\mathrm{ng} / \mathrm{ml})(\leq 78.4$ versus $>78.4)$ & 0.58 & 0.24 & - & - & 0.56 & 0.21 & - & - \\
\hline Gleason score $(\leq 7$ versus $\geq 8)$ & 0.65 & 0.38 & - & - & 0.59 & 0.24 & - & - \\
\hline Bone metastasis (negative versus positive) & 0.59 & 0.24 & - & - & 0.61 & 0.28 & - & - \\
\hline Lymph node metastasis (negative versus positive) & 0.71 & 0.6 & - & - & 0.74 & 0.56 & - & - \\
\hline Visceral metastasis (negative versus positive) & 0.53 & 0.13 & - & - & 0.52 & 0.089 & - & - \\
\hline
\end{tabular}

*Cut-off point of each continuous variable was the median value among the 74 patients included in this study. ADT: Androgen deprivation therapy; ECOG: Eastern Cooperative Oncology Group; LDH: lactate dehydrogenase; ALP: alkaline phosphatase; PSA: prostate-specific antigen.

conditions associated with cancer progression, such as high tumor cell turnover and normal tissue damage, are likely to elevate AST rather than ALT, thus making the De Ritis ratio a promising biomarker for malignant diseases. In fact, a number of studies reported the usefulness of the De Ritis ratio as a prognostic indicator for several types of malignant tumors $(8-12,21)$. As for prostate cancer, however, only the study by Wang et al showed that a higher De Ritis ratio could be predictive of a poorer pathological outcome and higher rate of biochemical recurrence in patients with localized disease (21), while the significance of this ratio in mCRPC patients remains unknown.

In this series, the median value of the De Ritis ratio in the 74 mCRPC patients included in this study was 1.35 , which was thus used as a cutoff point. According to this definition, the elevation of the De Ritis ratio in these 74 patients was significantly correlated with some characteristics suggesting an unfavorable clinical course, including an elderly age, higher LDH level, higher PSA level and presence of bone metastases. It may be difficult to theoretically elucidate the close association between the De Ritis ratio and conventional prognostic indicators in these $74 \mathrm{mCRPC}$ patients receiving cabazitaxel; however, these findings may be illustrated, at least in part, by the involvement of ALT rather than AST in aerobic glycolysis, which has been shown to be observed in actively proliferating cancer cells, including prostate cancer cells $(20,22,23)$.
To our knowledge, there have been limited studies assessing the value of a biomarker for mCRPC patients receiving cabazitaxel therapy. For example, Uemura et al. showed that the neutrophil-to-lymphocyte ratio (NLR) was significantly associated with the OS in $47 \mathrm{mCRPC}$ patients treated with cabazitaxel; however, there was no description of the predictive value of NLR for cabazitaxel therapy in these 47 patients (24). In the present study, therefore, the usefulness of De Ritis ratio as a predictive and/or prognostic biomarker for mCRPC patients receiving cabazitaxel therapy was investigated. Although there was no significant difference in the PSA response or PSA PFS on cabazitaxel therapy between the low and high De Ritis ratio groups, the OS in the high De Ritis ratio group was significantly poorer than that in the low ratio group. These findings suggest the utility of the baseline De Ritis ratio as a biomarker correlated with $\mathrm{mCRPC}$ patients receiving cabazitaxel therapy.

It is of interest to investigate the significance of the De Ritis ratio in relation to conventional prognostic parameters. In this series, despite the lack of a significant impact of the De Ritis ratio on the PSA PFS even on univariate analysis, this ratio, in addition to the ECOG PS and LDH level, was shown to be independently associated with the OS in the 74 mCRPC patients receiving cabazitaxel therapy on multivariate analysis. Considering the outcome described above as well as the convenient way of measuring the De Ritis ratio, which enables straightforward calculation based 
on the findings on routine laboratory examinations during the follow-up of mCRPC patients, it may be worthwhile to further address the role of the De Ritis ratio as one of the potential prognostic indicators in MCRPC patients who are scheduled to be treated with cabazitaxel.

Here, we would like to emphasize several limitations of this study. Firstly, this was a retrospective study with a comparatively small number of patients; thus, it is necessary to confirm the findings of this study in a prospective manner including a larger number of patients. Secondly, this study used the median value of the De Ritis ratio among the 74 included patients as its cut-off value; however, the optimal cutoff value of this ratio has not been established. Thirdly, only PSA PFS, but not radiographic PFS, was assessed in this study, since radiological examinations were performed based on the discretion of the treating physicians, resulting in the absence of these examinations for some patients even after PSA progression. Finally, due to the absence of widely accepted sequential therapy for mCRPC patients, the included patients in this study have a very heterogeneous character with respect to the previous history of systemic therapy prior to the introduction of cabazitaxel.

\section{Conclusion}

This is the first study to investigate the impact of the De Ritis ratio on the oncological outcomes following cabazitaxel therapy for mCRPC patients, and showed that the De Ritis ratio was significantly correlated with the OS, but not the PSA response or PSA PFS, in a total of 74 Japanese docetaxel-refractory mCRPC patients who were subsequently treated with cabazitaxel. Collectively, these findings suggest that the De Ritis ratio could be used as a prognostic, rather than predictive biomarker for mCRPC patients on cabazitaxel therapy; accordingly, the measurement of the De Ritis ratio may assist physicians in their decision-making concerning several issues related to cabazitaxel therapy, such as the indication, timing of induction and discontinuation of this agent.

\section{Conflicts of Interest}

The Authors declare that they have conflicts of interest regarding this study.

\section{References}

1 Mukherji D, Omlin A, Pezaro C, Shamseddine A and de Bono $\mathrm{J}$ : Metastatic castration-resistant prostate cancer (CRPC): preclinical and clinical evidence for the sequential use of novel therapeutics. Cancer Metastasis Rev 33: 555-566, 2014.

2 Fitzpatrick JM, Bellmunt J, Fizazi K, Heidenreich A, Sternberg CN, Tombal B, Alcaraz A, Bahl A, Bracarda S, Di Lorenzo G, Efstathiou E, Finn SP, Fosså S, Gillessen S, Kellokumpu-
Lehtinen PL, Lecouvet FE, Oudard S, de Reijke TM, Robson CN, De Santis M, Seruga B and de Wit R: Optimal management of metastatic castration-resistant prostate cancer: highlights from a European Expert Consensus Panel. Eur J Cancer 50: 16171627, 2014.

3 de Bono JS, Oudard S, Ozguroglu M, Hansen S, Machiels JP, Kocak I, Gravis G, Bodrogi I, Mackenzie MJ, Shen L, Roessner M, Gupta S and Sartor AO: Prednisone plus cabazitaxel or mitoxantrone for metastatic castration-resistant prostate cancer progressing after docetaxel treatment: a randomised open-label trial. Lancet 376: 1147-1154, 2010.

4 Fizazi K, Scher HI, Molina A, Logothetis CJ, Chi KN, Jones RJ, Staffurth JN, North S, Vogelzang NJ, Saad F, Mainwaring P, Harland S, Goodman OB Jr, Sternberg CN, Li JH, Kheoh T, Haqq $\mathrm{CM}$ and de Bono JS: Abiraterone acetate for treatment of metastatic castration-resistant prostate cancer: final overall survival analysis of the COU-AA-301 randomised, double-blind, placebocontrolled phase 3 study. Lancet Oncol 13: 983-992, 2012.

5 Scher HI, Fizazi K, Saad F, Taplin ME, Sternberg CN, Miller K, de Wit R, Mulders P, Chi KN, Shore ND, Armstrong AJ, Flaig TW, Fléchon A, Mainwaring P, Fleming M, Hainsworth JD, Hirmand M, Selby B, Seely L and de Bono JS: Increased survival with enzalutamide in prostate cancer after chemotherapy. N Engl J Med 367: 1187-1197, 2012.

6 Ozer J, Ratner M, Shaw M, Bailey W and Schomaker S: The current state of serum biomarkers of hepatotoxicity. Toxicology 245: 194-205, 2008.

7 De Ritis F, Coltorti M and Giusti G: An enzymic test for the diagnosis of viral hepatitis; the transaminase serum activities. Clin Chim Acta 2: 70-74, 1957.

8 Rawson NS and Peto J: An overview of prognostic factors in small cell lung cancer. A report from the Subcommittee for the Management of Lung Cancer of the United Kingdom Coordinating Committee on Cancer Research. Br J Cancer 61: 597-604, 1990.

9 Stocken DD, Hassan AB, Altman DG, Billingham LJ, Bramhall SR, Johnson PJ and Freemantle N: Modelling prognostic factors in advanced pancreatic cancer. Br J Cancer 99: 883-893, 2008.

10 Tan X, Xiao K, Liu W, Chang S, Zhang T and Tang H: Prognostic factors of distal cholangiocarcinoma after curative surgery: a series of 84 cases. Hepatogastroenterology 60: 18921895, 2013.

11 Bezan A, Mrsic E, Krieger D, Stojakovic T, Pummer K, Zigeuner R, Hutterer GC and Pichler M: The preoperative AST/ALT (De Ritis) ratio represents a poor prognostic factor in a cohort of patients with nonmetastatic renal cell carcinoma. J Urol 194: 30-35, 2015.

12 Nishikawa M, Miyake $\mathrm{H}$, Kurahashi $\mathrm{T}$ and Fujisawa $\mathrm{M}$ : Significance of multiple preoperative laboratory abnormalities as prognostic indicators in patients with urothelial carcinoma of the upper urinary tract following radical nephroureterectomy. Int J Clin Oncol 23: 151-157, 2018.

13 Scher HI, Halabi S, Tannock I, Morris M, Sternberg CN, Carducci MA, Eisenberger MA, Higano C, Bubley GJ, Dreicer R, Petrylak D, Kantoff P, Basch E, Kelly WK, Figg WD, Small EJ, Beer TM, Wilding G, Martin A and Hussain M: Design and end points of clinical trials for patients with progressive prostate cancer and castrate levels of testosterone: recommendations of the Prostate Cancer Clinical Trials Working Group. J Clin Oncol 26: 1148-1159, 2008. 
14 Eisenhauer EA, Therasse P, Bogaerts J, Schwartz LH, Sargent D, Ford R, Dancey J, Arbuck S, Gwyther S, Mooney M, Rubinstein L, Shankar L, Dodd L, Kaplan R, Lacombe D and Verweij J: New response evaluation criteria in solid tumours: revised RECIST guideline (version 1.1). Eur J Cancer 45: 228247, 2009.

15 Miyake H, Sakai I, Harada K, Muramaki M and Fujisawa M: Significance of docetaxel-based chemotherapy as treatment for metastatic castration-resistant prostate cancer in Japanese men over 75 years old. Int Urol Nephrol 44: 1697-1703, 2012.

16 Nozawa M, Mukai H, Takahashi S, Uemura H, Kosaka T, Onozawa Y, Miyazaki J, Suzuki K, Okihara K, Arai Y, Kamba T, Kato M, Nakai Y, Furuse H, Kume H, Ide H, Kitamura H, Yokomizo A, Kimura T, Tomita Y, Ohno K and Kakehi Y: Japanese phase I study of cabazitaxel in metastatic castrationresistant prostate cancer. Int J Clin Oncol 20: 1026-1034, 2015.

17 Miyake H, Sugiyama T, Aki R, Matsushita Y, Tamura K, Motoyama D, Ito T and Otsuka A: No significant impact of prior treatment profile with docetaxel on the efficacy of cabazitaxel in Japanese patients with metastatic castration-resistant prostate cancer. Med Oncol 34: 141, 2017.

18 Bando Y, Hinata N, Terakawa T, Furukawa J, Harada KI, Nakano $\mathrm{Y}$ and Fujisawa M: Activity of cabazitaxel in patients with metastatic castration-resistant prostate cancer after treatment with single or dual regimens of novel androgen receptor-targeting agents. Med Oncol 34: 163, 2017.

19 Seisen T, Rouprêt M, Gomez F, Malouf GG, Shariat SF, Peyronnet B, Spano JP, Cancel-Tassin G and Cussenot O: A comprehensive review of genomic landscape, biomarkers and treatment sequencing in castration-resistant prostate cancer. Cancer Treat Rev 48: 25-33, 2016.
20 vander Heiden MG, Cantley LC and Thompson CB: Understanding the warburg effect: the metabolic requirements of cell proliferation. Science 324: 1029-1033, 2009.

21 Wang H, Fang K, Zhang J, Jiang Y, Wang G, Zhang H, Chen T, Shi X, Li Y, Duan F and Liu J: The significance of De Ritis (aspartate transaminase/alanine transaminase) ratio in predicting pathological outcomes and prognosis in localized prostate cancer patients. Int Urol Nephrol 49: 1391-1398, 2017.

22 Tahir SA, Yang G, Goltsov A, Song KD, Ren C, Wang J, Chang W and Thompson TC: Caveolin-1-LRP6 signaling module stimulates aerobic glycolysis in prostate cancer. Cancer Res 73: 1900-1911, 2013.

23 Shiraishi T, Verdone JE, Huang J, Kahlert UD, Hernandez JR, Torga G, Zarif JC, Epstein T, Gatenby R, McCartney A, Elisseeff JH, Mooney SM, An SS and Pienta KJ: Glycolysis is the primary bioenergetic pathway for cell motility and cytoskeletal remodeling in human prostate and breast cancer cells. Oncotarget 6: 130-143, 2015.

24 Uemura K, Kawahara T, Yamashita D, Jikuya R, Abe K, Tatenuma T, Yokomizo Y, Izumi K, Teranishi JI, Makiyama K, Yumura Y, Kishida T, Udagawa K, Kobayashi K, Miyoshi Y, Yao $\mathrm{M}$ and Uemura $\mathrm{H}$ : Neutrophil-to-lymphocyte ratio predicts prognosis in castration-resistant prostate cancer patients who received cabazitaxel chemotherapy. Biomed Res Int 2017: 7538647, 2017.

Received May 17, 2018

Revised June 5, 2018

Accepted June 6, 2018 